\title{
THREADED FLUID CONDITION SENSOR FOR REAL-TIME, ON-LINE AND IN-LINE OIL CONDITION MONITORING
}

Andle, Jeffrey; Durdag, Kerem; Chap, Maly; and Haskell, Reichl

SenGenuity, a Division of Vectron Int'l

267 Lowell Rd., Hudson, NH USO 03051

\section{ABSTRACT}

Beginning January 1, 2007 on-highway diesel engines faced tougher emission standards for $\mathrm{NO}_{\mathrm{X}}$ and particulate matter. Over the course of the subsequent three years, $\mathrm{NO}_{\mathrm{x}}$ emissions will have trended down toward the 2010 standard, mentioned above. This phase-in provision allows the engine manufacturers to concentrate on reducing $\mathrm{NO}_{\mathrm{x}}$. On-highway fuel sulfur levels have dropped to $15 \mathrm{ppm}$, beginning in 2006 because even relatively small amounts of sulfur add to particulate exhaust emissions.

To achieve these emissions limits, OEMs are using a combination of cooled exhaust gas recirculation (EGR) at higher rates and exhaust after-treatment devices such as catalytic diesel particulate filters and oxidation catalysts. This has resulted in a new generation of engine oils that provide emission control system durability, prevent catalyst poisoning and particulate filter blocking, while still offering optimum protection for control of piston deposits, oxidative thickening, oil consumption, hightemperature stability, soot handling properties, foaming and viscosity consistency during shearing. These increased demands on the lubricant fluids require more accurate and reliable condition based monitoring.

There are two distinct drivers in the mobile asset on-highway (trucking and commercial automotive); 1) extending oil change intervals in commercial vehicles, and 2) optimizing and extending warranty on diesel engines in truck engines. In this document the focus is on the diesel engine market due to the urgency demonstrated by the market. Finally, it is important to note that for truck engine manufacturers, the requirements of any system for the determination of diesel oil quality is driven by their desire to rapidly and continuously monitor fuel dilution and soot loading (and possibly contaminants depending on usage conditions) in addition to traditional trend analysis of additives content (TBN, etc.).

To this end there is an unmet need for an in-line process monitoring device meeting the needs of the market. The present work shows progress towards a system that overcomes many of the limitations of prior sensor systems.

\section{INTRODUCTION}

There is no single measurement approach that is simultaneously suited to detecting all of the lubricant properties of interest, such as fuel dilution, soot loading, water and glycol contamination, additives depletion, and wear particle detection. The successful solution will include a fusion of sensor principles with the design and operation of the various subsystems properly integrated.

\section{Viscosity Measurement}

Viscosity measurements are critical to determining that the oil will provide the requisite lubrication function and are well suited to signaling the sudden onset of a catastrophic situation such as a fuel or coolant leak into the lubricant. Viscosity also indicates a change in base oil properties due to oxidation and other effects that occur after consumption of anti-oxidant additives. Because viscosity is a measure of serviceability of the oil as a lubricant, it is a critical measurement; however because non-catastrophic changes in viscosity occur after depletion of the protective additives, it is often argued that viscosity is a lagging indicator. Viscosity alone is also unable to distinguish fuel dilution from water or glycol contamination or soot loading from oxidation. Be that as it may, only continuous monitoring of viscosity can provide a reliable, immediate indicator of lubricant failure due to discrete, catastrophic events and viscosity data is important in confirming other so-called leading indicators. Viscosity measurement is a critical component of any multi-sensor suite.

A significant volume of work exists with regards to viscosity measurement of oils with an emphasis on in-engine measurements. Approaches suggested for the problem have been almost exclusively based on acoustic wave device (AWD) technology, in which the fluid in contact with a vibrating crystal results in changes in one or more electrical properties including wave velocity, resonant frequency, propagation loss or crystal resistance. The viscosity measurement is made by placing an AWD having a shear mode of vibration in contact with liquid. The liquid's viscosity and density determine the thickness of the fluid hydro-dynamically coupled to the surface of the sensor. The sensor surface is in 
uniform motion at frequency, $\omega=2 \pi F$, with amplitude, $U$. The frequency is known by design and amplitude is determined by the power level of the electrical signal applied to the sensor. As the shear wave penetrates into the adjacent fluid to a depth, $d$, determined by the frequency, viscosity and density of the liquid as $d=(2 \eta / \omega \rho)^{1 / 2}$. The shear rate is approximately $\omega U / d$.

Previous AWDs have included shear horizontal acoustic plate mode (SHAPM), shear horizontal surface acoustic wave (SH-SAW or Love Wave), thickness shear mode bulk acoustic wave (TSM BAW), and flexural tuning fork modes. These devices have all been resonant with frequencies of $100-160 \mathrm{MHz}$ typical of the SH-SAW and SHAPM devices, frequencies of $3-10 \mathrm{MHz}$ typical of the TSM devices and frequencies of 0.01 to $0.1 \mathrm{MHz}$ typical of the flexural devices.

Typical laboratory methods are linear (e.g. capillary) or rotational (e.g. spindle) and have a "frequency" of zero. The value of a truly Newtonian liquid is independent of frequency; however oils have both intermolecular friction and intramolecular compliance, resulting in a complex viscoelastic response. The result is Maxwellian behavior with a characteristic time constant, $\tau$. For mineral oils of moderately low viscosity the apparent relaxation times are on the order of 10 to $50 \mathrm{~ns}$. However, high molecular weight additives for viscosity index improvement can have time constants exceeding $1 \mu \mathrm{s}$. Good correlation to laboratory measurements appears to favor the flexural devices since they best exhibit $\omega \tau<1$. However, fluid condition monitoring seeks to measure the trending of a reproducible measurement and does not carry the burdens of quality control or research wherein the value itself is critical.

High Frequency AWD: Delay Line Sensors

High frequency devices such as the SHAPM at $160 \mathrm{MHz}{ }^{[1,2,3,4]}$ have been seen to be reproducible but are only marginally able to address the application. SH-SAW devices at $\sim 100 \mathrm{MHz}$ have enjoyed some commercial success and presumably employ correlation factors for a specific set of OEMapproved oils ${ }^{[5]}$. Both classes of devices have employed delay lines with separate input and output transducers separated by a propagation path. Simplistically the transducer properties can be assumed to be independent of loading and an increase in attenuation or an increase in delay time through the device occurs during fluid property change. The operating viscosity range of the sensors is readily able to cover the range of engine lubricants within the limitations of non-ideal responses caused by Maxwellian behavior. Another problem with these high frequency devices is the penetration depth. In water the penetration depth of a $160 \mathrm{MHz}$ sensor is on the order of $50 \mathrm{~nm}$. Even in a fluid with $\eta / \rho \sim 100 \mathrm{~mm}^{2} / \mathrm{s}$, like a heavy engine oil, the penetration depth is only $500 \mathrm{~nm}$. High molecular weight additives, used to modify viscosity index, are significant in size compared to this length scale.

\section{Low Frequency AWD: Flexural Tuning Fork Sensors}

Reasoning from the inverse, the lowest frequency devices have been suggested be the most desirable in order to maintain $\omega \tau<1$ and penetration depths large compared to molecular size ${ }^{[6]}$. At $\sim 32$ $\mathrm{KHz}$ the penetration depth into water is $3.5 \mu \mathrm{m}$ and it increases to $350 \mu \mathrm{m}$ in $100 \mathrm{~mm}^{2} / \mathrm{s}$ viscosity oils. While this improves the correlation to laboratory methods for clean and homogeneous solutions, the sensor becomes sensitive to particulates in suspension. Since the interaction is highly variable over different size and shape particulates, this can lead to irreproducible results.

The ideal size of a resonator at a given transducer impedance (crystal resistance) depends on the piezoelectric coupling. Quartz is almost universally favored for its environmental stability, low cost and extreme reproducibility; however it has poor piezoelectric coupling. Low frequency resonators, such as the flexural tuning forks, require large device sizes that are impractical in order to obtain low crystal resistance. Instead, designs use undersized resonators that physically meet the size requirements but then exhibit high resistance in air and even higher resistance in fluids. This is not a significant problem for hermetically sealed devices in frequency control applications with no fluid load since high impedance circuits are possible at such low frequencies with extremely low power consumption.

However, the devices are simple two-terminal resonators and therefore this crystal resistance is electrically in parallel with the transducer static capacitance. The reactance of the transducer static capacitance is comparable to the crystal resistance making measurements difficult. Since the crystal resistance increases with increasing fluid viscosity, there is an upper bound at which it becomes immeasurably large compared to the static capacitance's reactance.

In the case of tuning forks there exists the further complication that the electrodes are directly wetted and the added conductance and capacitance of the fluid is in shunt with the resonance, making measurements in contaminated oils unreliable. This arrangement, inherent to the device structure, provides serious limitations to measurement range and also on the accuracy of the interrelated measurements. The use of materials with significantly higher piezoelectric coupling is unlikely to be successful since these materials are pyroelectric and are ferroelectric, having higher dielectric constants than quartz-like materials, interfering with conductivity and dielectric measurements. 
Mid Frequency AWD: TSM Resonator Sensors

Thickness shear mode (TSM) technology offers a useful trade-off between the viscosity range of delay lines and the more Newtonian sensing mechanisms of the low frequency resonators. Prior efforts to employ TSM devices wetted both electrodes, incurring similar issues as were observed in the low frequency tuning forks. Others packaged the sensor to wet only one surface. Nonetheless, in various early implementations the wetted electrode was not grounded or a low precision circuit was frequently used. Again, the low piezoelectric coupling of quartz was a significant obstacle.

The monolithic crystal filter (MCF) was introduced by Schweyer et al. ${ }^{[7,8]}$ in 1997 as a solution to the circuit limitations of single pole TSM devices. The structure uses two TSM resonant structures in such close proximity to form a single device having a three-terminal, two-port topology. The measurand is the transfer resistance between the input and output electrodes while the electrode capacitances are between these electrodes and ground. Performance was still limited by piezoelectric coupling; however the limitation was scaled to higher viscosities. The development of lanthanum gallium silicate (LGS) allowed successful devices to be demonstrated in $2007^{[9]}$. Related structures for high pressure operation were shown in $2008^{[10]}$.
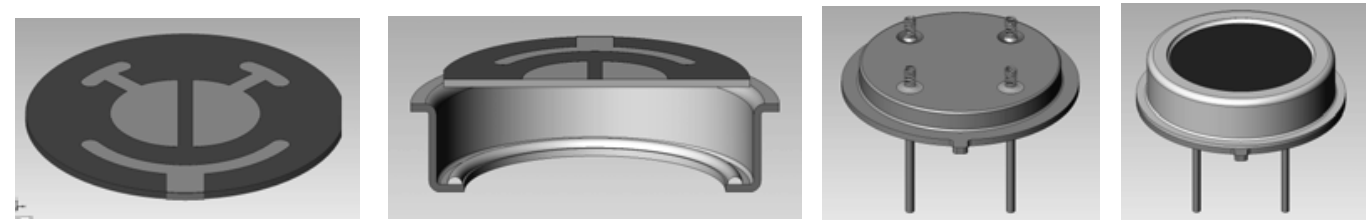

Figure 1. Packaging of the langasite MCF sensor starts with a polyimide wrap of the ground contact (left) followed by epoxy die attach (middle left). The package is affixed with springs (middle right) that connect to the sensors pads via conductive epoxy. The sensor element is vacuum baked and then weld sealed (right).

\section{Electrical Measurements}

While viscosity indicates the current condition of the oil, electrical measurements such as conductivity and dielectric or infrared spectroscopy measurements can probe the chemical nature. Each contaminant has a unique infrared (IR) absorption signature. There has been limited success in applying IR spectroscopy to used oils since the broad and substantial absorption due to soot quickly overshadows the other spectra and seriously limits the penetration depth of IR signals through the oil.

Instead, conductivity and dielectric measurements are preferred. Water and glycol cause significant increases in the dielectric constant (capacitance) of the oil and also tend to increase conducivity. Soot loading also affects the dielectric and conductivity, however with a different frequency and temperature dependence. A significant body of technical literature and patent history applies to the design and operation of conductivity/dielectric (C/D) electrodes in oil.

Generally the dielectric constant of base oil is low and is stable over a wide range of measurement frequencies from $\ll<1 \mathrm{MHz}$ to $\sim 10 \mathrm{MHz}$. Increases in dielectric constant due to water loading, for example, lower the upper corner frequency, which is determined by parasitic inductance of the measurement cell. Increases in conductivity decrease the RC time constant of the sample and raise the lower corner frequency.

The conductivity of pure base oils can be very low, on the order of $1000 \mathrm{pS} / \mathrm{m}$. Reliable measurements of such low conductivities require low frequencies to avoid being loaded by the capacitive reactance but cannot employ DC measurement due to surface effects in the fluid. Frequencies of 0.01 $\mathrm{Hz}$ to $1 \mathrm{~Hz}$ appear to be best for this measurement.

\section{RECENT RESULTS}

The present work seeks to merge C/D measurement of electrical properties and the most recent MCF sensor technology with temperature correction circuitry and digital electronics to provide a complete, multi-measurand, smart sensor for fluid condition monitoring.

\section{Viscosity Sensing Elements}

The viscosity sensing element, shown in Figure 1, exhibits a well defined insertion loss ranging from nearly $0 \mathrm{~dB}$ (unity transfer) in air to well over $-40 \mathrm{~dB}(10 \mathrm{mV} / \mathrm{V})$ in saturating viscosity fluids. The insertion loss is equivalent to a series transfer resistance that ranges from less than $100 \Omega(-6 \mathrm{~dB})$ to more than $10 \mathrm{~K} \Omega(-40 \mathrm{~dB})$. The resistance in air, $R_{a}$, is due to circuit and packaging parasitics and the intrinsic losses of the crystal. Additional equivalent resistance is incurred in proportion to the square root of viscosity, such that $R=R_{a}+S_{v}(\rho \eta)^{1 / 2}$ for dynamic viscosity $\eta(\mathrm{mPa})$ and density $\rho\left(\mathrm{g} / \mathrm{cm}^{3}\right)$. The 
frequency in air at $25^{\circ} \mathrm{C}$ is also analyzed. Individual manufacture with shadow masks introduces $\sigma \sim 8 \%$ in the sensitivity, necessitating sensor calibration.
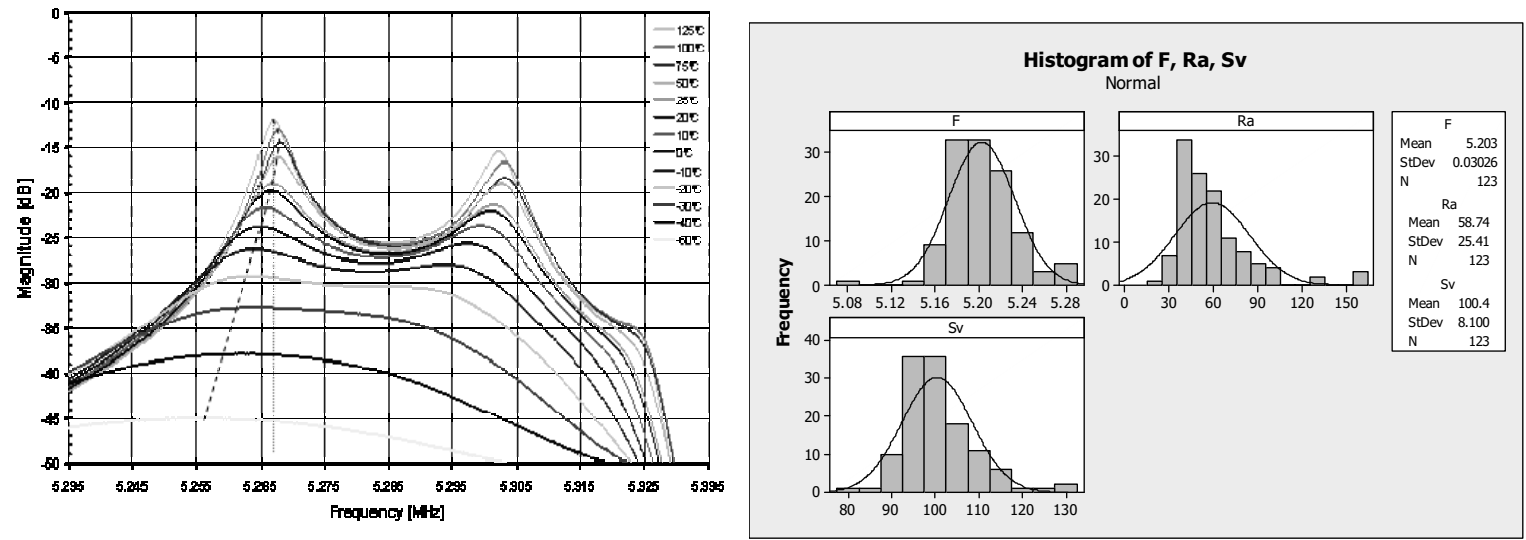

Figure 2. (Left) Response of an MCF coupled resonator in $\mathrm{HT}-270$ perfluoropolyether from $125^{\circ} \mathrm{C}$ (minimum loss curve) to $-40^{\circ} \mathrm{C}$. The dotted line indicates the resonant peak measured by oscillator circuitry. (Right) Statistical analysis of 123 sensor elements in air $\left(F, R_{a}\right)$ and their sensitivity $\left(S_{v}\right)$ shows good reproducibility.

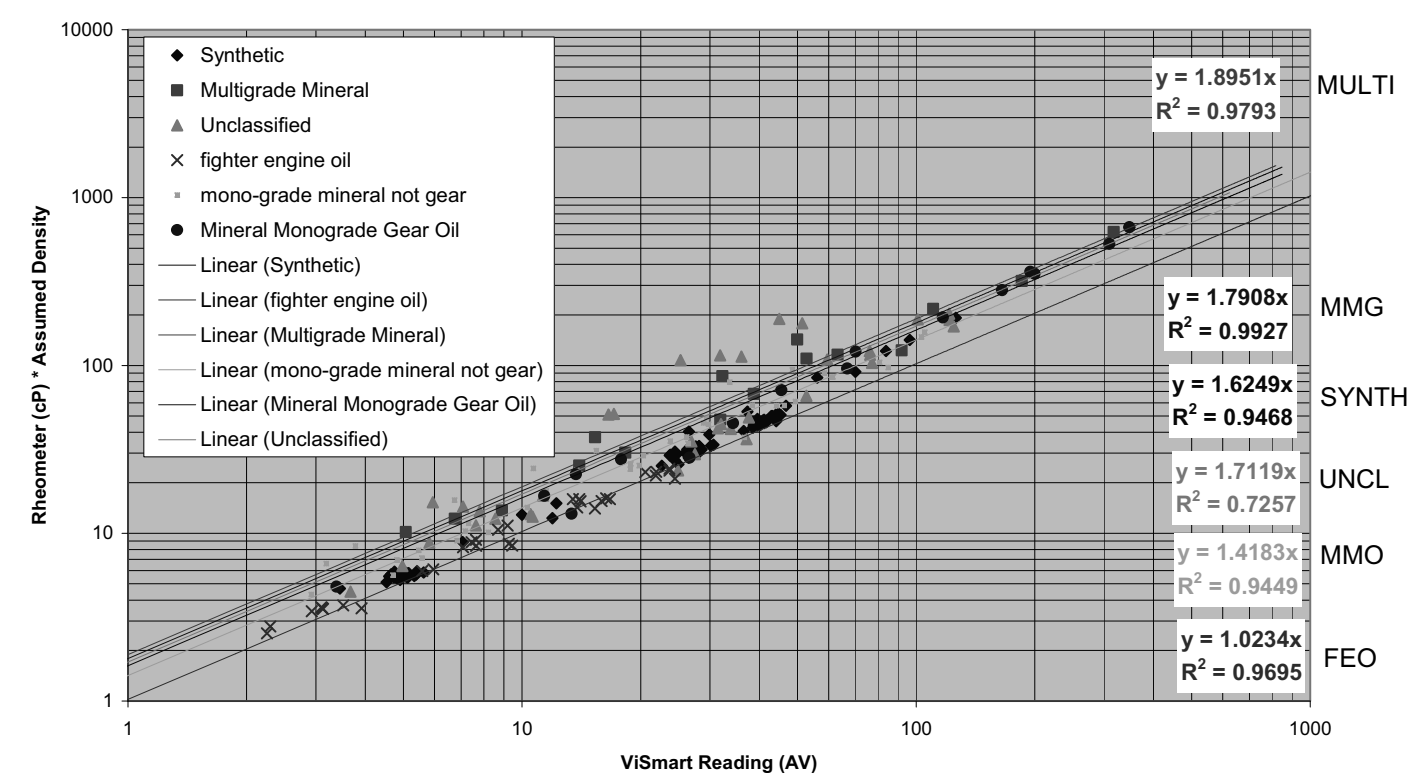

Figure 3. Linear correlations of several classes of engine oil, turbine oil, and hydraulic fluids. Good correlation is seen over temperature and aging of mono-grade and multi-grade oils, both synthetic and mineral within any given classification. Unclassified oils show considerable remaining variance.

Viscosity Measurement System and Results

The sensor element has traditionally been incorporated into a feedback oscillator with diode detection circuitry and temperature measurement. The insertion loss is approximated as the voltage transfer function measured by the two diode detector circuits as the oscillator freely tracks the sensor (e.g. dotted line of Figure 2). The result is a commercially available viscosity-only sensor product.

Correlation of the resulting viscometer to lab data for numerous oils was undertaken and is summarized in Figure $3^{[11,12]}$. The goal of the product characterization was to create a set of fluid-specific and class-specific correlations between the lab-measured kinematic viscosity $(n / \rho)$ at low shear rate and linear flow, as employed in ASTM and MIL specifications of a lubricant, with the measured "acoustic viscosity" native to the sensor under test $(\rho \eta)$. The conversion of acoustic viscosity data into kinematic viscosity at least requires multiplication by the square of the density $\left(\mathrm{g} / \mathrm{cm}^{3}\right)$. A nominal density of 0.88 yields a nominal conversion factor of 0.7744 . The correlation is further complicated by harmonic motion at $2 \pi^{\star} 5.3 \times 10^{6}$ radians per second in the vibration based measurement and an estimated shear rate of 
the acoustic viscometer between 4,000 and 40,000 1/s. No significant dependence on shear rate was observed in lab measurements and the factor was completely attributed to Maxwellian effects of the viscoelastic fluids at the moderately high resonant frequencies.

\begin{tabular}{|l|c|c|c|c|l|}
\hline & Slope & Exp & R2 & Tau (ave) ns & \\
\hline Default Fit & 1.1544 & 1.0422 & $94.18 \%$ & varies & \\
\hline Fighter Engine & 1.0234 & 1 & $96.95 \%$ & 30.2 & default density may be low \\
\hline Mono-grade Mineral not Gear & 1.4183 & 1 & $94.49 \%$ & 35.5 & hydraulic, turbine, transmission and diesel \\
\hline Synthetic (other) & 1.6249 & 1 & $94.68 \%$ & 38.0 & All synthetics but fighter engine oil and SHC 639 \\
\hline Unclassified & 1.7119 & 1 & $72.57 \%$ & 39.0 & MIXED \\
\hline Mono-grade Mineral Gear Oil & 1.7908 & 1 & $99.27 \%$ & 39.9 & \\
\hline Multi-Grade mineral & 1.8951 & 1 & $97.93 \%$ & 41.1 & Gear and Diesel multigrades \\
\hline
\end{tabular}

Table I. Power law and linear fits are listed for all fluids and for classes of fluids. The $R^{2}$ values are generally good except for the mixed (unclassified) oils.

\section{Conductivity-Dielectric Electrode Design}

Historical approaches to $C / D$ electrodes have included surface interdigital electrodes for high cell constant and concentric electrodes for self cleaning within the flow of a tube or pipe and for total containment of fringing fields. Surface electrodes are particularly sensitive to surface contamination by soot. Initially the approach taken was to place an outer electrode concentric with the metal transistor outline (TO) package of the sensor. This approach was guided by the conventional wisdom that concentric electrodes were superior. It was quickly determined that this geometry exacerbated fouling for a threaded sensor and further that it was difficult to control dimensions.

Until recently the approach typically taken by OEMs had been to place the sensor into the oil pan. This was found to result in unacceptable fouling rates, especially given the new regulations and the increased soot collection. Instead, the sensor should be bolted into the rifle of the diesel engine and subjected to high flow rates parallel to the surface. In the present work a parallel plate electrode structure is formed with the plates parallel to the laminar flow of lubricant in the engine as seen in Figure 4.
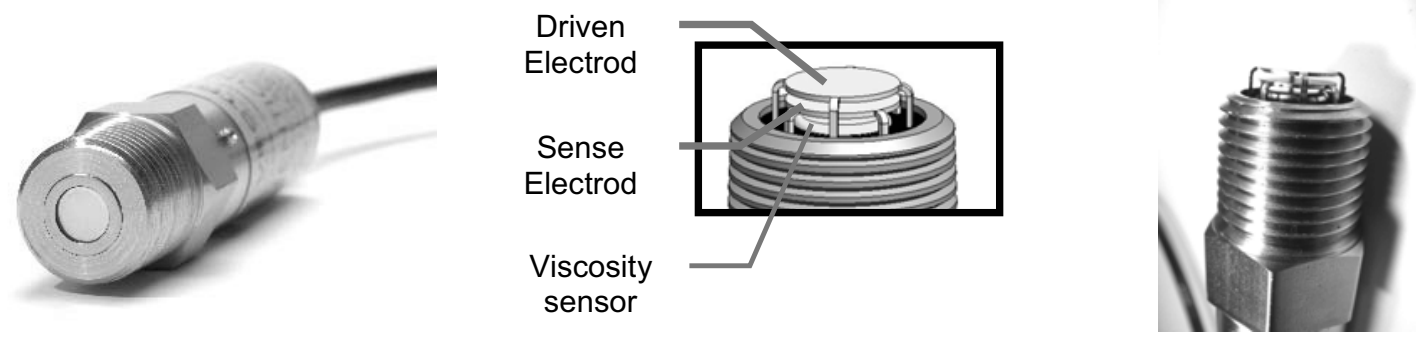

Figure 4. (Left) The existing viscosity-only sensor is to be modified to integrate conductivity-dielectric electrodes. (Middle) Schematic of the integrated conductivity-dielectric electrode over the viscosity sensor in a threaded multi-measurand sensor. (Right) In the resulting system, a platinum RTD is housed in the viscosity sensor package.

Cell capacitance was measured in the lab using RF transmission in an Agilent 8753C network analyzer. Admittance parameters were calculated from the instrument and $Y 21$ was interpreted as a lossy capacitance. It was found that $3 \mathrm{MHz}$ measurements gave the best immunity from stray inductance and leakage currents in the various liquids. Cell capacitance is measured in air and in deionized water to obtain the parasitic capacitance $(2.5 \mathrm{pF})$ and the cell constant $(0.155 \mathrm{~m})$. Measurements at $40^{\circ} \mathrm{C}$ and $90^{\circ} \mathrm{C}$ showed no significant temperature dependence of the calibration parameters.

Conductance was measured using a Keithley $6517 \mathrm{~B}$ electrometer to output a $\pm 1 \mathrm{~V}$ square wave with 50 s periodicity $(0.02 \mathrm{~Hz})$ and to measure the correlated current averaged after $20 \mathrm{~s}$ from each step edge. Dividing by the cell constant yields conductivity in $\mathrm{ps} / \mathrm{m}$.

\section{CONCLUSIONS AND FUTURE WORK}

The effort to date has clearly shown that the viscosity-only sensor and instrumentation give reproducible and reliable measurement with simple correlation to lab measured kinematic viscosity. The resulting sensor can address the viscosity measurement requirements of a more complete fluid condition sensor. Improvements to pressure handling and to circuit optimization are underway.

The effort to date has demonstrated an integrated $C / D$ electrode set that is compatible with the fluid flow dynamics of the rifle in a diesel engine. The electrodes are readily integrated into the threaded sensor format and their construction is consistent with the wide operating range requirements of the application. Future work will refine and integrate sensor electronics to replace the lab instruments employed in the present effort. 


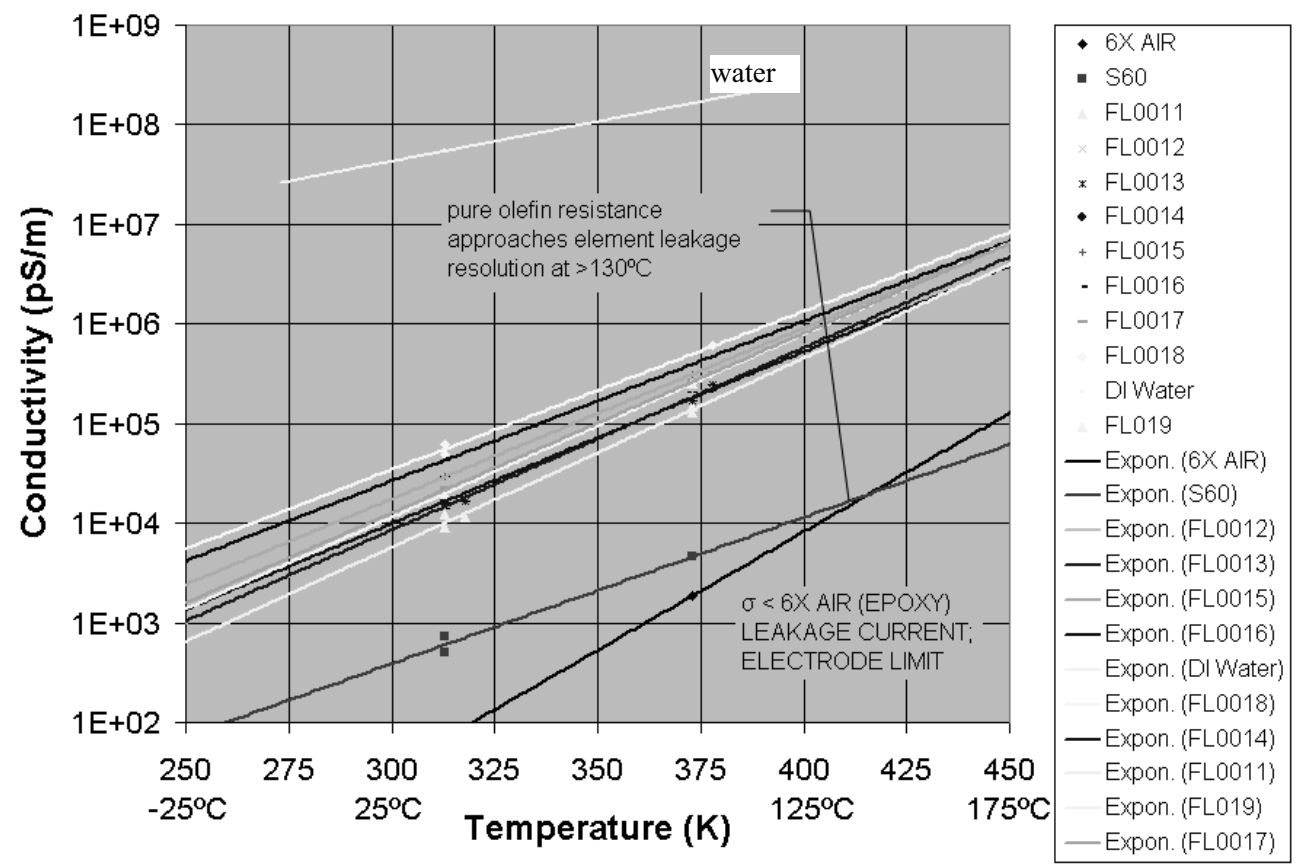

Figure 5. Conductivity vs. temperature data is presented for several oils including a pure polyolefin (S60) and also for deionized water. The data indicates that cell construction does not limit the performance.

\section{ACKNOWLEDGEMENTS}

The authors would like to acknowledge Shunhe Xiong for help in developing the correlations of the oil samples and Johnson Uch for taking data on the sensing elements.

\section{REFERENCES}

[1] A.J. Ricco and S.J. Martin, “Acoustic wave viscosity sensor”, Appl. Phys. Lett. 50, 1474 (1987).

[2] S. Martin, A. Ricco, T. Niemczyk and G. Frye, "Characterization of SH acoustic plate mode liquid sensors", Sensors \& Actuators B: 20, 253-268 (1989) and references therein.

[3] J. J. Height, D. Huynh, M. G. Schweyer, J. T. Weaver, J. C. Andle, D. J. McAllister and J. F. Vetelino, "Comparison of surface transverse wave (STW) and shear horizontal acoustic plate mode (SHAPM) devices for biochemical sensors", 1997 IEEE Int'1 Frequency Control Symposium, pp. 147-155.

[4] J. Andle, R. Haskell, R. Sbardella, G. Morehead, M. Chap, J. Columbus and D. Stevens, "Design, Optimization and Characterization of an Acoustic Plate Mode Viscometer", 2007 IEEE Sensors Symposium.

[5] Herrmann F.; Hahn D.; Buttgenbach S.; "Separate determination of liquid density and viscosity with sagittally corrugated Love-mode sensors", Sensors and Actuators A: Physical, Volume 78, Number 2, 14 December 1999, pp. 99-107(9).

[6] L.F. Matsiev, J.W. Bennett, E.W. McFarland, "Application of Low Frequency Mechanical Resonators to Liquid Property Measurements". Proceedings of 1998 IEEE Ultrasonics Symposium. v.1, 459-462

[7] M. Schweyer, J. Hilton, J. Munson, J. Andle, J. Hammond and R. Lec, “A novel monolithic piezoelectric sensor”, 1997 IEEE International Frequency Control Symposium, pp. 32-40.

[8] M. Schweyer, J. Hilton, J. Munson, J. Andle, J. Hammond, R. Lec \& Q. Lin, “A Novel Monolithic Piezoelectric Sensor”, 1997 IEEE International Ultrasonics Symposium, pp. 371-374.

[9] J. Andle, R. Haskell, R. Sbardella, G. Morehead, M. Chap, J. Columbus and D. Stevens, "Design, packaging and characterization of a two-port bulk wave langasite viscometer", IEEE Sensors 2007.

[10] J. Andle, R. Haskell and M. Chap, "Electrically Isolated Thickness Shear Mode Liquid Phase Sensor for High Pressure Environments", 2008 IEEE Ultrasonics Symposium (in press).

[11] K. Durdag and J. Andle, "Portable/Handheld Oil Assessment Device Project: NCMS Collaborative Agreement No: 200640-140414", Final Report, Nov. 12, 2007 (unpublished).

[12] K. Durdag and J. Andle, "Real-Time Viscosity Measurement for Condition-Based Monitoring Using Solid-State Viscosity Sensor", Tribology Transactions, 51:296-302, 2008. 